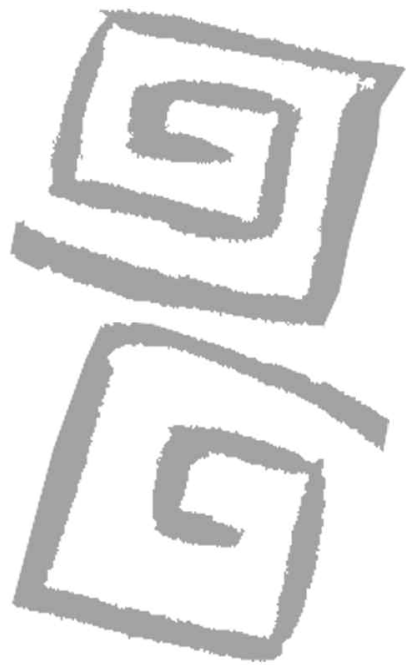

${ }^{1}$ Licenciada en Economía. Magíster en Finanzas Públicas Provinciales y Municipales. Directora de la Fundación Mundo Sano, Argentina. starragona@mundosano.org

${ }^{2}$ Licenciada en Economía. Doctora en Economía. Investigadora, Centro de Investigaciones y Estudio sobre Cultura y Sociedad (CIECS), Consejo Nacional de Investigaciones Científicas y Técnicas (CONICET), Universidad Nacional de Córdoba (UNC), Argentina. montemale@yahoo.com

${ }^{3}$ Licenciada en Economía. Consultora, Fundación Mundo Sano, Argentina. marchioni.silvia@gmail.com

${ }^{4}$ Licenciado en Economía. Investigador, Instituto de Efectividad Clínica y Sanitaria (IECS), Argentina.

joaquincaporale@gmail.com

${ }^{5}$ Médica. Especialista en Pediatría. Especialista en Salud Pública. Responsable del área médica, Fundación Mundo Sano, Argentina. apereiro@mundosano.org

${ }^{6}$ Médico. Investigador Instituto de Investigaciones de Enfermedades Tropicales (IIET), Argentina. palaciosmaxi@gmail.com

\section{Dengue en la Argentina: un análisis económico del impacto de la epidemia de 2009}

\author{
Dengue in Argentina: an economic analysis of the \\ impact of the 2009 epidemic
}

Tarragona, Sonia'; Monteverde, Malena²; Marchioni, Silvia3; Caporale, Joaquín ${ }^{4}$; Pereiro, Ana Cristina ${ }^{5}$; Palacios, Julio Maximiliano ${ }^{6}$

RESUMEN El presente estudio estima la carga económica de los casos notificados de dengue durante la epidemia argentina de 2009. Entre los costos considerados, se encuentran los costos médicos para el tratamiento de las personas afectadas y los costos de oportunidad de aquellas personas que dejaron de trabajar o estudiar a causa de la enfermedad. Para la determinación de los costos, a fines de 2009 se llevó a cabo una encuesta ad hoc a 201 hogares en los que habitaban personas que habían padecido la enfermedad durante la epidemia. Los resultados obtenidos permiten estimar entre los 26 y los 40 millones de pesos (entre 6 y 9 millones de dólares) el costo total de los casos notificados. Las regiones del norte argentino son las que habrían soportado la mayor carga económica (más del 90\% de la carga total). Para todas las regiones, el costo social de un caso de dengue representaría más del $40 \%$ de la remuneración neta mensual promedio de los individuos.

PALABRAS CLAVE Dengue; Costo de Enfermedad; Argentina.

ABSTRACT The present study estimates the economic burden associated with the cases of dengue recorded during the 2009 Argentine epidemic. Among the costs considered are the medical costs necessary for the treatment of those affected and the opportunity costs for those who stopped working or studying because of the disease. In order to assess the costs of the disease, at the end of 2009 an ad-hoc survey was carried out in 201 households where people that had contracted dengue during the 2009 epidemic lived. The results allow us to estimate between 26 to 40 million Argentine pesos (6 to 9 million US dollars) as the total cost of the recorded cases. The regions in the north of Argentina bore the highest economic burden of the disease (more than $90 \%$ of the total burden). For all regions, the social cost of one dengue case accounts for more than $40 \%$ of the average monthly net individual income.

KEY WORDS Dengue; Cost of Illness; Argentina. 


\section{INTRODUCCIÓN}

La incidencia del dengue ha crecido dramáticamente alrededor del mundo en las últimas décadas y se estima que cerca de 2.500 millones de personas se encuentran en riesgo, lo que equivale a aproximadamente dos quintos de la población mundial. La Organización Panamericana de la Salud estima que cada año se notifican 50 millones de infecciones por dengue en el mundo (1).

Esta enfermedad es endémica en más de 100 países en África, América, el sudeste asiático y el oeste del Pacífico. En la actualidad, no solo se está experimentando un crecimiento en el número de casos, sino que también se están observando brotes explosivos con mayor frecuencia (2). Los países de la región más afectados son: Brasil, Ecuador, Colombia, Paraguay y Venezuela (3).

El brote que enfrentó la Argentina en el año 2009 significó la peor epidemia de dengue de su historia, tanto por la cantidad de personas afectadas como por la dispersión geográfica de los casos autóctonos que excedieron los canales endémicos tradicionales. De acuerdo a cifras oficiales, se notificaron algo más de 27.000 casos y las provincias más afectadas fueron Chaco (42\% de los casos), Catamarca (33\%), Salta (11\%), Jujuy (5\%), Santa Fe (4\%) y Tucumán (2\%), aunque también fueron registrados casos autóctonos en la Ciudad Autónoma de Buenos Aires, Misiones y Entre Ríos. Las provincias del norte argentino son las de mayor riesgo: solo las provincias de Chaco, Catamarca y Salta concentraron alrededor del $86 \%$ de los casos de dengue autóctonos registrados durante la primera mitad de 2009 (4).

El presente estudio busca aproximar la carga económica agregada asociada a los casos de dengue ocurridos durante la epidemia de Argentina a lo largo de la primera mitad del año 2009. En la estimación se incluyen los costos de los servicios médicos necesarios para el tratamiento de las personas afectadas por la enfermedad, así como el costo de oportunidad de aqueIlas personas que dejaron de realizar sus tareas formales habituales (como trabajar o estudiar) a causa del padecimiento de la enfermedad. Para la estimación de los costos se ha optado por emplear una perspectiva social, es decir, en términos de lo que le cuesta a la sociedad en su conjunto, sin diferenciar sobre quién recaen dichos costos (los pacientes, el Estado, las familias, etc.).

No se incluyen estimaciones de costos de las medidas de prevención de nuevos casos, implementadas con posterioridad al inicio de la epidemia (ni aun aquellas practicadas durante o con anterioridad a la misma), ni las pérdidas que la misma pudo ocasionar sobre actividades económicas específicas (como el turismo) o sobre el crecimiento económico agregado de la región o del país afectado (que podrían ser objeto de otros estudios).

\section{MATERIALES}

\section{Cantidades}

La estimación de costos se realizó a partir de la información sobre "cantidades" de bienes y servicios consumidos, extraída de una encuesta ad hoc realizada durante los meses de octubre y noviembre de 2009 en las zonas afectadas. Dicha encuesta permitió conocer la duración promedio de la enfermedad (distinguiendo entre los casos ambulatorios y hospitalizados), las frecuencias de uso de bienes y servicios médicos, y las frecuencias relativas a los costos de oportunidad (días de escolaridad y días de trabajo perdidos) para una muestra de los casos de dengue ocurridos durante el primer semestre de 2009. El universo poblacional objeto de análisis fueron las personas que padecieron la enfermedad durante la epidemia de 2009 en Argentina. Sin embargo, dadas las restricciones para realizar una encuesta con alcance en todo el territorio del país y por la inexistencia de registro de los domicilios de las personas afectadas, la selección de la muestra se basó en una población de una región endémica de la Argentina, crítica en términos de la presencia de casos de dengue durante la epidemia, donde además se verificó un registro exhaustivo de los domicilios de las personas afectadas que permitió el contacto posterior. Dado lo anterior, se seleccionó la ciudad de Orán en la provincia de Salta, una de las ciudades con mayor número de casos de la provincia y una de las más afectadas del país, cuya distribución por grupos de 
edad es similar a la distribución etaria para el total del país. La muestra quedó constituida por todos los casos de dengue oficialmente notificados ocurridos en la ciudad de Orán durante la epidemia. Cabe resaltar que, en esta ciudad, se contaba con información precisa del lugar de residencia de todos los casos notificados (no así para la totalidad de los casos en el país). Así, se identificaron 201 casos de dengue notificados oficialmente en la ciudad de Orán durante el período bajo análisis, a los que se visitó y se obtuvo información a partir de un cuestionario estructurado de preguntas. En la determinación de las proporciones y cantidades de servicios e insumos empleados se adoptó el supuesto de que, en todos los casos atendidos en efectores públicos, se siguieron rigurosamente los protocolos de atención recomendados por la autoridad sanitaria nacional, lo que permite suponer que no deberían esperarse variaciones significativas en la atención brindada en cualquier otro lugar del país.

\section{Precios}

Con el objetivo de minimizar los problemas de valoración asociados con la existencia de subsidios, se utilizó información de precios de los bienes y servicios médicos (medicamentos, prácticas de laboratorio, honorarios médicos y hospitalización) en el mercado privado, siempre que fue posible. Dadas las diferencias de precios y salarios existentes a lo largo del territorio argentino, se buscó recabar información representativa para cada una de las regiones afectadas.

Para el caso de los medicamentos (paracetamol e ibuprofeno) se consideran tanto los precios sugeridos de venta al público vigentes a nivel nacional (que coinciden con los precios de mercado), según información del Manual Farmacéutico (5), como los precios promedio de licitaciones realizadas por el Ministerio de Salud de la Nación para estos medicamentos distribuidos a través del mayor programa de provisión pública de medicamentos (Programa Remediar). Dado que se desconoce la proporción de población que fue tratada en el sector público en relación con las que recibieron medicamentos por parte del sector privado, se suponen dos escenarios extremos: uno en el que todos los medicamentos utilizados son de provisión privada (a precios de venta al público) y otro en el que todos son de provisión pública (a precios de las licitaciones públicas). En la práctica, tanto los precios pagados por el Estado, como los precios de venta al público no presentan diferenciales significativos entre regiones $y / 0$ entre provincias y por tanto se considera un precio único promedio para todo el territorio nacional, en cualquier caso. A su vez, los precios por unidad del paracetamol y del ibuprofeno muestran una considerable dispersión hacia el interior del segmento, dependiendo de la marca comercial, la presentación del producto y la cantidad de unidades por envase en los casos de los precios de venta al público, y también variaciones entre las distintas licitaciones públicas por lo que se trabajó con precios unitarios promedios.

Para las prácticas de laboratorio, se consideraron los precios que actualmente enfrenta el subsistema de Seguridad Social, con base en la información de la Confederación Unificada Bioquímica de la República Argentina y según información de laboratorios privados.

Las consultas médicas y las hospitalizaciones se valuaron de acuerdo a los precios provistos por diferentes centros de salud pertenecientes al subsector privado de las provincias de Chaco, Salta y Buenos Aires. En el caso de las hospitalizaciones, además se consideraron precios desde diversos nomencladores utilizados por las instituciones provinciales del seguro de salud.

Por su parte, para la asignación de un valor a las pérdidas de días de escuela, se sigue la metodología de estudios similares y se considera que el valor económico de un día de escuela es al menos igual al costo de proveer un día de escuela en el sistema público (6) y, siendo conservadores, el mismo se estima sobre la base del costo de la matrícula escolar de las escuelas públicas. A su vez, el costo de la matrícula escolar se estima a partir de la información de los presupuestos provinciales destinados a educación y al número de matrículas de todos los niveles educativos de las provincias para el año 2008, última información disponible (7-13). Para la actualización de los costos al año 2009, se realizó un ajuste de los mismos por el Índice de Precios al Consumidor nivel general (14). A su vez, para calcular los costos promedio por matrícula se tuvieron en cuenta los meses lectivos y los días 
hábiles, considerando que dicho período corresponde al ciclo escolar. Para estos cálculos se han seleccionado seis jurisdicciones, ya que constituyen lugares de referencia para las tres regiones bajo estudio y, a su vez, se trata de las jurisdicciones donde se presentó la mayor cantidad de casos de dengue durante 2009. Ellas son: Chaco (región Noroeste), Salta y Catamarca (región Nordeste), y Buenos Aires, Ciudad de Buenos Aires y Santa Fe (región Centro).

Por último, el costo de oportunidad de los días de trabajo perdidos se estima a partir de información de salarios por jurisdicción (para Chaco, Salta, Catamarca, Buenos Aires, Ciudad de Buenos Aires y Santa Fe) para el año 2009, según cifras del Ministerio de Economía y Finanzas Públicas (15).

\section{MÉTODOS}

Con el objeto de estimar la carga económica de la enfermedad, se aproximan ecuaciones de costos individuales promedio para distintas regiones de Argentina, que se combinan con información oficial de casos de dengue notificados para la obtención de los costos totales por región y para el total país.

La estimación del costo médico individual promedio para cada región surge de la siguiente expresión:

$C M_{i}=\left(P_{i}^{M} \times F^{M} \times \operatorname{Pr}^{M}\right)+\left(P_{i}^{V} \times F^{V} \times \operatorname{Pr}^{V}\right)+\left(P_{i}^{I} \times F^{I} \times \operatorname{Pr}^{I}\right)+\left(P_{i}^{L} \times F^{L} \times \operatorname{Pr}^{L}\right)$

\section{Donde:}

$\mathrm{CM}_{\mathrm{i}}$ es el costo médico promedio para un individuo en presencia de la enfermedad en la región $\mathrm{i} ; \mathrm{P}_{\mathrm{i}} \mathrm{M}$ es el precio diario del medicamento para la región i (precio unitario del medicamento multiplicado por la dosis diaria promedio); $\mathrm{F}^{\mathrm{M}}$ es la frecuencia promedio de uso de medicamentos (en días), entre los casos que usaron medicamentos, según información obtenida de encuesta ad hoc; $\mathrm{Pr}^{\mathrm{M}}$ es la proporción de casos que usó medicamentos para el tratamiento, según encuesta ad hoc; $\mathrm{P}_{\mathrm{i}} \mathrm{V}$ es el precio promedio de una consulta médica para la región $\mathrm{i} ; \mathrm{F}^{\mathrm{V}}$ es la frecuencia promedio de visitas médicas, entre los que realizaron consultas, según encuesta ad hoc; $\operatorname{Pr}^{\mathrm{V}}$ es la proporción de casos que consultó al médico, según encuesta ad hoc; $\mathrm{P}_{\mathrm{i}}^{\mathrm{I}}$ es el precio promedio de un día de internación para la región i; FI es la frecuencia promedio de internación (en días), según encuesta ad hoc; $\operatorname{Pr}^{\mathrm{I}}$ es la proporción de casos de internación, según encuesta ad hoc; $\mathrm{P}_{\mathrm{i}} \mathrm{L}$ es el precio promedio de los estudios de laboratorio para la región i; $\mathrm{FL}^{\mathrm{L}}$ es la frecuencia promedio de estudios de laboratorio según estándares médicos (medido en cantidad de estudios estándar para un caso de dengue) y; $\operatorname{Pr}^{\mathrm{L}}$ es la proporción de casos con síntomas de la enfermedad para aquellos para los que fueron realizados análisis de laboratorio.

Por su parte, la estimación de los costos de oportunidad promedio para un individuo en presencia de la enfermedad en cada región sigue la expresión de la siguiente ecuación:

$$
C O_{i}=\left(P_{i}^{S} \times F^{S} \times \operatorname{Pr}^{S}\right)+\left(P_{i}^{E} \times F^{E} \times \operatorname{Pr}^{E}\right)
$$

Donde:

$\mathrm{CO}_{\mathrm{i}}$ es el costo de oportunidad promedio en la región $\mathrm{i} ; \mathrm{P}_{i} \mathrm{~S}$ es el salario promedio por día para la región i (y según la distribución por edad, sexo y nivel de educación de los casos de dengue en cada región); $\mathrm{F}^{\mathrm{S}}$ es la frecuencia de días laborales perdidos por la enfermedad, según encuesta ad hoc (se incluye los días perdidos por el paciente y sus cuidadores); $\operatorname{Pr}^{S}$ es la proporción de pacientes con dengue y de cuidadores que tuvieron que faltar al trabajo, según encuesta ad hoc (ajustado por la tasa de desempleo de cada región); $\mathrm{P}_{\mathrm{i}} \mathrm{E}^{\mathrm{e}} \mathrm{es}$ el precio de un día de escuela perdido en la región $\mathrm{i} ; \mathrm{F}^{\mathrm{E}}$ es la frecuencia de días de escuela perdidos por la enfermedad, según encuesta ad hoc $y ; \operatorname{Pr}^{E}$ es la proporción de casos de dengue que debieron faltar a la escuela, según encuesta ad hoc (ajustado por la estructura de edades de los casos registrados en cada región).

\section{RESULTADOS}

\section{Encuesta}

De acuerdo a los resultados de la encuesta, el $91 \%$ de los casos con dengue notificados fueron pacientes ambulatorios y el $9 \%$ requirieron hospitalización. Entre los casos 
ambulatorios, la duración promedio de la enfermedad fue de 10 días, mientras que entre los hospitalizados la duración promedio fue de casi 18 días. Los pacientes hospitalizados indicaron que, en promedio, estuvieron algo más de 5 días internados.

El $83 \%$ de los pacientes ambulatorios indicó haber tomado medicación y el $85 \%$ indicó haber realizado al menos una visita médica. El $83 \%$ de los pacientes, que tomó medicación, indicó haberlo hecho durante 7 días en promedio y el $85 \%$, que indicó haber ido al médico, realizó en promedio 3 visitas.

El análisis de la pérdida de días de trabajo y de días de escuela, se realizó entre las personas que indicaron que trabajaban y/o estudiaban al principio de la encuesta. Entre las personas que al principio de la encuesta indicaron que trabajaban, el 82\% (de los que respondieron) indicó que debió faltar a su trabajo durante el período de enfermedad. Entre los que faltaron al trabajo, lo hicieron en promedio 8 días. Por su parte, entre las personas que estudiaban, un $80 \%$ de los casos (que respondieron) indicó que debió faltar a la escuela y, entre ellos, la pérdida de días de escuela en promedio fue de casi 9 días.

\section{Costos}

La estimación de los costos médicos se realizó bajo tres escenarios según el tipo de servicio utilizado. El primer escenario asume la utilización de las alternativas médicas menos costosas, mientras que el tercer escenario, las más caras.

Dado que el costo de los medicamentos representa entre el $0,2 \%$ y el $1 \%$ del costo promedio individual total para cada escenario (según se utilice precio pagado por el Estado o precio de venta al público), solo se presentan en el texto de este documento los resultados obtenidos en el caso de que los medicamentos empleados hayan sido valorizados a precio pagado por el Estado. La comparación con el precio de venta al público se incluye en los cuadros incluidos en este apartado. Cabe aclarar que todas las equivalencias en dólares estadounidenses incluidas en el texto corresponden al tipo de cambio oficial de julio de 2009 (4,55 pesos por dólar).

En el primer escenario se asume que las personas que consumieron medicamentos tomaron paracetamol, los casos de internación se suponen en sala general y el test de laboratorio utilizado para la detección de dengue es el IgM o el lgG. De acuerdo a dicho escenario, los costos médicos individuales en promedio serían de 592 pesos (130 dólares) para la región Centro (Ciudad Autónoma de Buenos Aires y provincias de Buenos Aires, Córdoba, Entre Ríos, La Pampa y Santa Fe), 409 pesos (90 dólares) para las regiones del Noroeste Argentino (NOA) y la región de Cuyo (Catamarca, La Rioja, Jujuy, Salta, Santiago del Estero, Tucumán, Mendoza, San Juan y San Luis) y de 466 pesos (102 dólares) para la región del Noreste Argentino (NEA) (Chaco, Corrientes, Formosa y Misiones).

Para el segundo escenario se asume que la droga utilizada fue ibuprofeno, los casos de internación fueron en sala de cuidados especiales y el test de confirmación de dengue fue obtenido a partir de la utilización de PCR. Según dicho escenario, los costos individuales promedio serían de: 864 pesos (190 dólares) en la región Centro, 600 pesos (132 dólares) en las regiones del NOA y Cuyo, y 680 pesos (149 dólares) en la región del NEA.

El tercer escenario difiere del segundo solamente en el tipo de internación: en lugar de sala de cuidados especiales, supone la internación en terapia intensiva. En dicho caso, los costos individuales promedio ascenderían a 1.473 pesos (324 dólares) en la región Centro, 714 pesos (157 dólares) en las regiones del NOA y Cuyo, y 1.138 pesos (250 dólares) en la región del NEA.

Para la estimación de la proporción de personas afectadas por la pérdida de días de trabajo (como consecuencia de la enfermedad), se ajustaron los valores obtenidos en la encuesta (la proporción de personas que debieron dejar de trabajar) de acuerdo a la proporción de la población afectada en cada región en edades activas y por la tasa de desempleo de cada región en el año 2009 de acuerdo con la información oficial $(4,16)$, en virtud de que una persona afectada por la enfermedad, que no trabaja por razones voluntarias o se encuentra desempleada, no sufre un costo de oportunidad asignable a la enfermedad.

Las estimaciones de los costos de oportunidad por pérdida laboral indican que, en promedio, un individuo de la región Centro dejó de percibir (o de generar) 542 pesos (119 dólares) como consecuencia del estado de enfermedad, y 
dicha cifra sería de 499 (110 dólares) y de 468 pesos (103 dólares) para los individuos de las regiones del NOA y Cuyo y del NEA respectivamente.

Por su parte, para la estimación de la proporción de la población que sufrió un costo de oportunidad por pérdida de escolaridad, se ajustaron los resultados obtenidos en la encuesta (personas que estudiaban y que debieron faltar a la escuela por la enfermedad) a partir de información sobre el grupo de edad al que pertenecen las personas afectadas en cada región (4), asumiendo que la pérdida de escolaridad se puede dar en el rango de los 5 a los 24 años de edad.

Las estimaciones de costos de oportunidad (individuales promedio) por pérdida de escolaridad arrojan los siguientes valores: 57 pesos para la región Centro (13 dólares), 40 pesos (9 dólares) para las regiones del NOA y Cuyo y 52 (11 dólares) para la región del NEA.

En el Cuadro 1 se presentan los costos promedios individuales utilizados como base para el cálculo de los costos médicos, los costos de oportunidad y el costo individual total. Además, se presenta la información de precios y cantidades utilizada para estimar los mismos:

a) En la primera columna se describen los distintos conceptos que componen los costos. En el caso de los costos médicos, se muestran las distintas alternativas de medicamentos y servicios de cada uno de los escenarios antes descritos.

b) La segunda, tercera y cuarta columnas muestran la información sobre "cantidades", es decir, la proporción de población a la que se aplica cada concepto y la frecuencia de uso de cada bien, servicio o días de escuela o de trabajo perdidos, cuando se trata de costos de oportunidad. En el caso de los medicamentos y la consulta médica, los conceptos se aplican exclusivamente en los casos ambulatorios (ya que en los costos de internación, estos se contemplan dentro del costo por día de internación). Los casos ambulatorios representan el $91 \%$ de los casos de la muestra (proporción 1), lo que se multiplica por la proporción de casos que utilizó medicamentos y realizó consulta médica (proporción 2) y la cantidad de días de uso de medicamentos y cantidad de consultas médicas (frecuencias), según los resultados de la encuesta. De forma similar se procede con los casos de internación y con las pruebas de laboratorio. Para el cálculo del costo de oportunidad por días de trabajo perdidos se multiplica la proporción 2, correspondiente a la razón entre las personas de la encuesta que respondieron que tuvieron que dejar de trabajar como consecuencia de la enfermedad (y el total de personas que trabajaba), por la proporción 1 que, a su vez, es el producto de: i) la población afectada por la enfermedad en edades activas en cada región (según información oficial) y ii) (1- tasa de desempleo/100) de cada región (según información oficial). Por tanto, la proporción 1 en este caso se utiliza para ajustar por los diferenciales entre regiones tanto de composición etaria de la población afectada como de desempleo de cada región. Para el costo de oportunidad por pérdida de escolaridad, se multiplica la proporción 1, correspondiente a la razón entre la cantidad de personas en el grupo de 5 a 24 años afectadas y el total de personas afectadas en cada región (de acuerdo a información oficial) y la segunda proporción, que corresponde a las personas encuestadas que respondieron que habían perdido días de escuela entre las que respondieron que asistían a la escuela al principio de la encuesta.

c) Las columnas 5, 6 y 7 muestran los precios unitarios de los bienes y servicios médicos y de trabajo y los días de escuela, para cada región. Cabe aclarar que el precio de los medicamentos está expresado en días (donde la dosis por día es de tres pastillas de paracetamol 500 mg o Ibuprofeno $400 \mathrm{mg}$ ).

d) Finalmente, las columnas 8, 9 y 10 muestran los costos promedios individuales para cada concepto, incluidos los subtotales para los costos médicos y los costos de oportunidad y los costos promedios individuales totales (que son la suma de los dos subtotales).

Cabe señalar que los costos individuales así estimados son la suma de promedios ponderados, donde el peso de cada concepto está dado por el perfil de las personas afectadas en la muestra, en términos de la severidad del caso (ambulatorio o de internación), el tipo de bien o servicio médico utilizado, la edad y la actividad de la persona afectada. Es decir, si bien el modelo de 


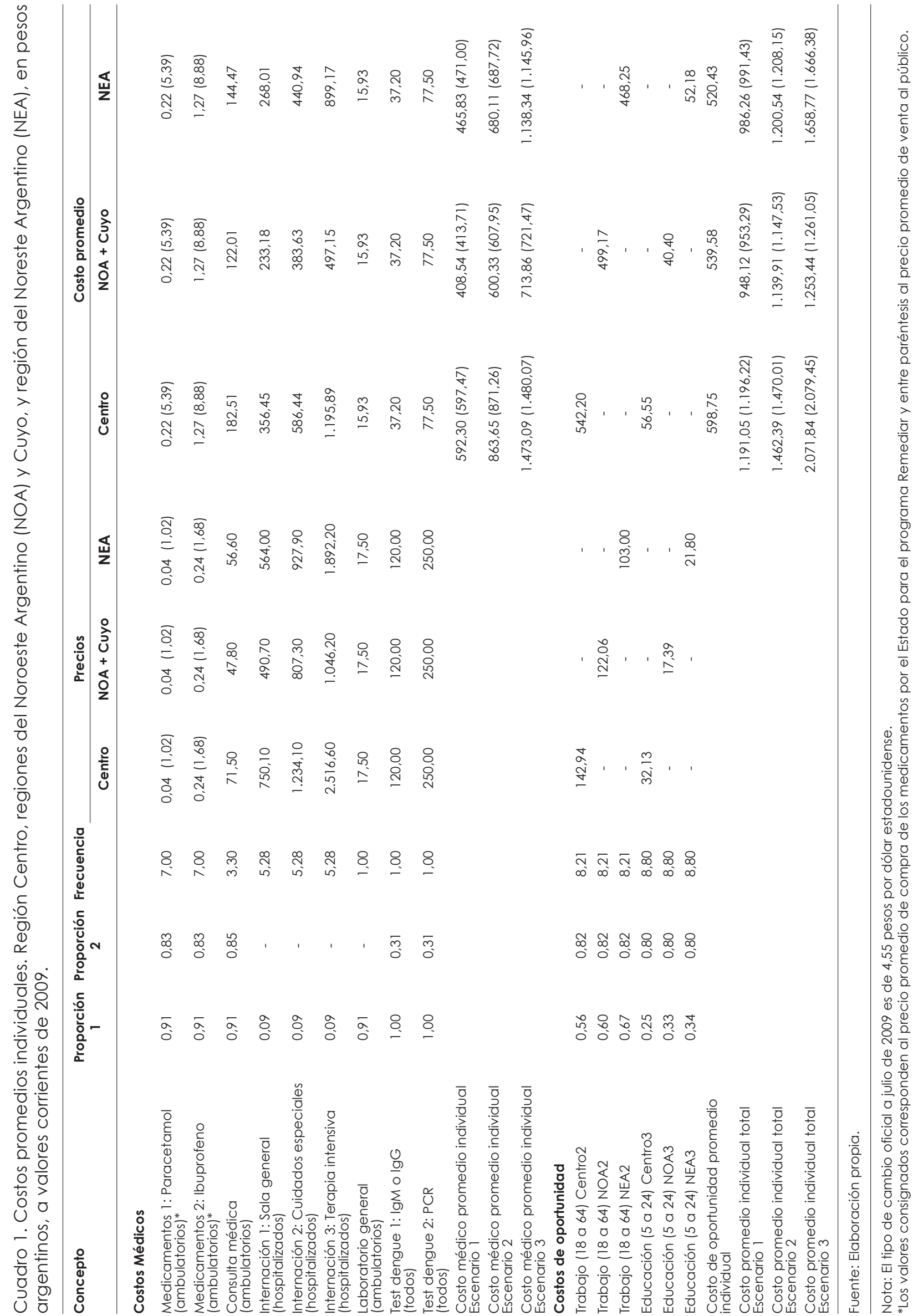


estimación se basa en costos promedio (que se aplican a todos los casos de dengue oficialmente notificados), en el cálculo se toma en cuenta el peso relativo de los distintos "perfiles" o tipos de casos y los costos asociados a dichos perfiles, lo que permite ponderar el hecho de que los costos de un grupo de pacientes no necesariamente sigue una distribución normal (porque un pequeño número de casos puede representar un muy alto costo en términos del tratamiento médico o la duración del tratamiento que necesita, mientras que la mayoría de los casos pueden ser pacientes con costos de tratamiento mucho más bajos).

Con el objeto de evaluar en qué medida las pérdidas de ingresos y los gastos en tratamientos implicarían una carga económica significativa para las familias -en el caso de que tuvieran que afrontarlos ciertamente-, se estimó la relación entre los costos individuales promedio de la enfermedad en cada región (costos médicos y de oportunidad) y la remuneración neta mensual promedio en las mismas regiones (15).

Los resultados de estimar dicho cociente son representados en la Figura 1 donde se muestra que para todas las regiones y bajo los

Figura 1. Incidencia del costo individual del dengue en la remuneración neta mensual para la región Centro, la región del Noreste Argentino (NEA) y las regiones del Noroeste Argentino (NOA) y Cuyo. 2009.

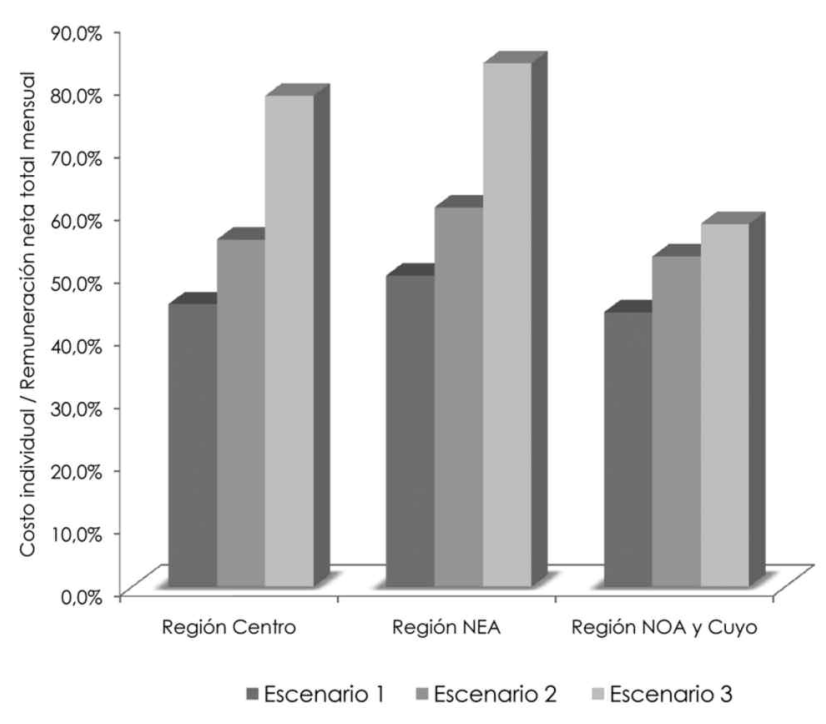

Fuente: Elaboración propia. tres escenarios de atención médica considerados, el costo de un caso de dengue representa más del $40 \%$ de la remuneración neta mensual promedio de los individuos. La región con la relación costo/remuneración neta mensual más elevada es la región del NEA, le sigue la región Centro y por último las regiones del NOA y Cuyo.

En el Cuadro 2 se presentan los resultados de los costos totales para cada región y para el total del país para los tres escenarios evaluados. Según dichos resultados, la epidemia de dengue ocurrida en la Argentina en el año 2009, habría implicado un costo para el total de la economía argentina que oscilaría entre los 26 millones y los 40 millones de pesos argentinos (entre 6 y 9 millones de dólares, según el tipo de cambio vigente en el año 2009).

\section{DISCUSIÓN}

El presente estudio aproximó, por primera vez para la Argentina, la carga económica asociada a los casos de dengue oficialmente notificados durante la epidemia de 2009.

La estimación de los costos totales arrojó como resultado que la epidemia de dengue del año 2009 habría implicado una carga económica para la economía argentina que oscilaría entre los 26 millones y los 40 millones de pesos a valores corrientes (entre 6 y 9 millones de dólares), según el escenario de atención médica.

La importancia relativa de los costos médicos y de los costos de oportunidad sería similar, aunque varía según sea el escenario de servicios médicos considerados. En el primer escenario (en el que se asume el uso de los servicios médicos menos costosos), los costos médicos representarían entre el $43 \%$ y el $50 \%$ de la carga económica total según la región (Centro, Noreste y Noroeste más Cuyo). En el segundo escenario dicha importancia relativa oscilaría entre el $53 \%$ y $59 \%$ y, en el tercer escenario, entre el $57 \%$ y el $71 \%$, según la región.

Las regiones del norte argentino son las que habrían soportado la mayor carga económica de la enfermedad (con más del 90\% de la carga total del país), a pesar de los menores costos unitarios de los servicios médicos y de oportunidad. Si 
Cuadro 2. Costo económico del dengue para la región Centro, las regiones del Noroeste Argentino (NOA) y Cuyo, la región del Noreste Argentino (NEA) y para el total país.

\begin{tabular}{|c|c|c|c|c|c|}
\hline Región & $\begin{array}{l}\text { Provincias de la } \\
\text { región afectadas }\end{array}$ & $\begin{array}{l}\text { Casos } \\
\text { totales }\end{array}$ & $\begin{array}{c}\text { Costo total } \\
\text { Escenario } 1 \text { (en \$) }\end{array}$ & $\begin{array}{c}\text { Costo total } \\
\text { Escenario } 2 \text { (en \$) }\end{array}$ & $\begin{array}{c}\text { Costo total } \\
\text { Escenario } 3 \text { (en \$) }\end{array}$ \\
\hline Centro & $\begin{array}{l}\text { Capital Federal, } \\
\text { Provincia Buenos Aires, } \\
\text { Córdoba, Entre Ríos, } \\
\text { Santa Fe }\end{array}$ & 1.625 & $1.935 .458(1.943 .861)$ & $2.376 .392(2.388 .763)$ & $3.366 .738(3.379 .110)$ \\
\hline NOA y Cuyo & $\begin{array}{l}\text { Catamarca, La Rioja, } \\
\text { Jujuy, Salta, Santiago } \\
\text { del Estero y Tucumán. } \\
\text { Mendoza y San Luis }\end{array}$ & 14.238 & 13.499 .290 (13.572.912) & $16.230 .070(16.338 .469)$ & 17.846 .442 (17.954.842) \\
\hline NEA & $\begin{array}{l}\text { Chaco, Corrientes y } \\
\text { Misiones }\end{array}$ & 11.180 & 11.026 .428 (11.084.237) & $13.421 .990(13.507 .108)$ & $18.545 .062(18.630 .180)$ \\
\hline Total país & & 27.043 & $26.461 .176(26.601 .010)$ & $32.028 .451(32.234 .341)$ & $39.758 .242(39.964 .132)$ \\
\hline
\end{tabular}

Fuente: Elaboración propia.

Nota: Los valores consignados corresponden al precio promedio de compra de los medicamentos por el Estado para el programa Remediar y entre paréntesis al precio promedio de venta al público, ambos expresados en pesos argentinos. El tipo de cambio oficial a julio de 2009 es de 4,55 pesos por dólar estadounidense.

bien el número de casos de la región Centro se incrementó durante la última epidemia, casi el $94 \%$ de los casos ocurrieron en el norte de país explicando la alta carga económica para la región.

Para todas las regiones y bajo los tres escenarios de atención médica considerados, el costo de un caso de dengue representa más del $40 \%$ de la remuneración neta mensual promedio de los individuos. La región con la relación costoremuneración neta mensual más elevada es la región del NEA, le sigue la región Centro y por último las regiones del NOA y Cuyo.

Entre las limitaciones del estudio se encuentra la falta de información para estimar costos no médicos tales como costos de transporte y otros costos asociados con permanecer lejos del lugar de residencia como consecuencia de la enfermedad. Tampoco se consideraron las pérdidas que la epidemia de dengue pudo haber ocasionado sobre actividades económicas específicas (como el turismo) o sobre el crecimiento económico agregado de la región afectada.

Por otra parte, dado que el objetivo inicial del estudio fue estimar la carga económica de la enfermedad asociada específicamente al número de casos de dengue ocurridos durante la epidemia de 2009, y no la carga económica de la enfermedad de dengue de forma más general, no se incluyen los costos de las acciones de prevención de la enfermedad.

Las estimaciones de cantidades (frecuencias de uso de servicios y de días de escuela y de trabajo perdidos) se basan en información para una muestra de una población específica (la población de la ciudad de Orán, en la provincia de Salta). Para las estimaciones de costos se utilizan las frecuencias promedio estimadas con base en la muestra y se asume que las mismas son representativas de las frecuencias promedio de la población afectada en todo el país. Por tanto, la extrapolación implica suponer que los promedios de duración de la enfermedad, la frecuencia de uso de bienes y servicios médicos y los promedios de día de escuela y trabajo perdidos son relativamente constantes entre regiones. Si bien dicho supuesto puede parecer fuerte, la evidencia existente para algunas de esas cantidades indica que las mismas son relativamente constantes, incluso entre poblaciones muy diferentes entre sí. De acuerdo a estimaciones de Suaya et al. (15), la duración promedio de la enfermedad, entre los casos ambulatorios, oscila entre los $9 y$ 
los 12 días (un solo país de los ocho países de América Latina y Asia analizados en el estudio registró una duración promedio más elevada: 19,6 días para Panamá), lo que resulta muy similar a lo estimado en el presente estudio con base en la información muestral (que arrojó 10 días en promedio de duración). Para los casos de dengue hospitalizados, los promedios del trabajo de Suaya et al. indican que la duración de la enfermedad oscila en general entre los 10 y los 19 días, mientras que las estimaciones basadas en la muestra para Orán arrojan una duración promedio de casi 18 días.

Por último, para la estimación del valor de un día de escuela perdido, se siguió la metodología utilizada por Suaya et al. (15), donde se considera el valor de la matrícula escolar. Tal estimación del valor de un día de escuela puede considerarse una aproximación indirecta del costo económico (de oportunidad) de los recursos ya invertidos que dejan de utilizarse, pero no aproximan el valor presente de los salarios que pudieran verse afectados si la pérdida de días de escuela impactara en alguna medida sobre la productividad futura de los estudiantes afectados.

En todos los países en los que se han presentado brotes de dengue hay evidencia de que las cifras oficiales subestiman el verdadero número de casos, lo que pone de manifiesto la necesidad de considerar factores de expansión que permitan realizar estimaciones más aproximadas (17). Según estudios previos, se estima que por cada caso de dengue hospitalizado hay entre 1,6 a 3,2 casos que también debieron haber recibido dicha atención pero no la recibieron, y para los casos de dengue ambulatorio se estima que existen entre 10 a 27 casos sin diagnóstico por cada caso diagnosticado (18-20). Para el total de casos se estima que, en promedio, por cada caso notificado existen 6 casos no detectados por las estadísticas sanitarias oficiales (21). En consecuencia, los resultados obtenidos en el presente estudio resultan ciertamente conservadores, dado que solo se han considerado los casos oficialmente reportados. En efecto, si se contemplaran tres casos no registrados por cada caso notificado, las estimaciones alcanzarían a una carga económica que oscilaría entre 78 y 120 millones de pesos (17 y 26 millones de dólares) y si se consideraran los estudios previos mencionados estas cifras se posicionarían entre los 160 y 240 millones de pesos (35 y 53 millones de dólares).

Los resultados obtenidos muestran la pesada carga que significó la epidemia de dengue ocurrida en la Argentina en el año 2009 para el sistema de salud y para la sociedad en su conjunto.

Se está experimentando un crecimiento en el número de casos y en la frecuencia de los brotes en el mundo y en la región. De los 250.000 casos registrados en los primeros años de la década de 1990 en la región de las Américas, se reportaron 600.000 a principios de la actual década y solo en el 2007 se registraron más de 890.000 casos, de los cuales 26.000 fueron casos de dengue hemorrágico (2).

Dada la tendencia actual y ante la falta de campañas efectivas de prevención, cabe esperar que el dengue sea una enfermedad en aumento en la Argentina. Se sabe que en aquellas personas infectadas por una cepa, que contraen dengue nuevamente por una cepa diferente, el riesgo de muerte se incrementa. Frente a esta situación, la falta de efectivas campañas de prevención no solo implicaría un aumento en el número potencial de casos afectados sino también un incremento en la probabilidad de casos más severos, aumentando no solo la carga económica de la enfermedad sino la carga de enfermedad en términos de mayor riesgo de discapacidad y mortalidad de la población afectada.

Los resultados muestran, además, que los estudios sobre carga económica deberían contemplar un espectro amplio de los costos generados por la enfermedad. Los costos médicos implicados en el tratamiento de las personas afectadas representan una fracción importante de la carga total del dengue, pero no necesariamente la porción más importante. El costo de oportunidad asociado a la pérdida de días laborables y días de escuela representa otra fracción importante de la carga económica de la enfermedad. 


\section{AGRADECIMIENTOS}

Agradecemos al equipo de agentes de atención primaria de la localidad de Orán por su importante colaboración en la realización de las encuestas. En especial se agradece al Dr. Luis Mario Arias (Director del Hospital San Vicente de Paul de San Ramón de la Nueva Orán) y al Dr. Pedro Cortada (Director de Epidemiología del mismo hospital). El estudio fue realizado gracias al apoyo financiero y a la iniciativa de la Fundación Mundo Sano. Se agradecen también los comentarios y las sugerencias realizadas por los revisores de la revista Salud Colectiva que sin duda han ayudado a mejorar el documento original.

\section{REFERENCIAS BIBLIOGRÁFICAS}

1. San Martín JL, Brathwaite O, Zambrano B, Solórzano JO, Bouckenooghe A, Dayan GH, Guzmán MG. The epidemiology of dengue in the Americas over the last three decades: A worrisome reality. American Journal of Tropical Medicine and Hygiene. 2006;82(1):128-135.

2. WHO. Dengue and severe dengue: Fact sheet No117. [Internet] WHO; 2012 [citado 8 mar 2012]. Disponible en: http://www.who.int/mediacentre/ factsheets/fs117/en.

3. Torres J. El dengue en América Latina: ¿una situación única? [Internet] Universidad Central de Venezuela; 2002 [citado 6 abr 2010]. Disponible en: http://caibco.ucv.ve/caibco/ vitae/VitaeDieciocho/Articulos/Infectologia/Arch ivosHTML/dengue.pdf.

4. Ministerio de Salud de la Nación. Planilla de Notificación de Casos de Dengue, 1 de junio de 2009. Buenos Aires: MSAL; 2009.

5. Alfabeta. Informes de precios online a octubre 2009 [Internet]. 2009 [citado 15 feb 2010]. Disponible en: http://www.alfabeta.net/home/.

6. Suaya JA, Shepard DS, Siqueira JB, Martelli CT, Lum LSC, Tan LH, et al. Cost of dengue cases in eight countries in the Americas and Asia: A prospective study. American Journal of Tropical Medicine and Hygiene. 2009;80(5):846-855.

7. Gobierno de la Provincia de Catamarca. Ley de Presupuesto 2008 [Internet]. Catamarca: Ministerio de Hacienda y Finanzas; 2008 [citado 5 feb 2010]. Disponible en: http://www.hacienda.catamarca.gov.ar/normativas/Ley $\% 20$ de $\% 20 p$ resupuesto $\% 202008$.pdf.

8. Gobierno de la Provincia de Salta. Ley No 7.486, Ley de Presupuesto de la Provincia de Salta, Ejercicio 2008 [Internet]. Salta: Ministerio de Finanzas y Obras Públicas; 2008 [citado 5 feb
2010]. Disponible en: http://presupuesto.salta. gov.ar/Leyes/Textos/2008/2008\%20$\%$ 20Ley\%207.486.pdf.

9. Gobierno de la Provincia de Chaco. Ley $N^{\circ}$ 6.089 [Internet]. Chaco: Ministerio de Economía, Producción y Empleo; 2008 [citado 5 feb 2010]. Disponible en: http://economia.chaco.gov.ar /index.php? option $=$ com_wrapper\&view $=$ wrapper\&ltemid $=201$.

10. Gobierno de la Ciudad de Buenos Aires. Ley No 2.571, Presupuesto para el año 2008 [Internet]. Ciudad Autónoma de Buenos Aires: Hacienda; 2008 [citado 5 feb 2010]. Disponible en: http://estatico.buenosaires.gov.ar/areas/hacienda/pr esupuesto2008/pdf/ley2571/ley2571.pdf.

11. Gobierno de la Provincia de Buenos Aires. Ley 13.786, Presupuesto General [Internet]. Provincia de Buenos Aires: Ministerio de Economía; 2008 [citado 5 feb 2010]. Disponible en: http://www.ec. gba.gov.ar/areas/hacienda/Presupuesto/Presupuest os/2008/php/ejercicio2008.php.

12. Gobierno de Santa Fe. Ley $N^{\circ} 12.850$ [Internet]. Santa Fe: Sistema de Información Normativa; 2007 [citado 5 feb 2010]. Disponible en: http://gobierno.santafe.gov.ar/sin/mitemplate.php?tiponorma $=$ ley\&anio norma $=2007 \&$ nro ley $=12850 \&$ fecha norma $=27 / 12 / 2007$.

13. Dirección Nacional de Información y Evaluación de la Calidad Educativa. Anuario Estadístico Educativo 2009 [Internet]. Ministerio de Educación de la Nación [citado 10 feb 2010]. Disponible en: http://diniece.me.gov.ar/index.php? option $=$ com_content $\&$ task $=$ category \&sectio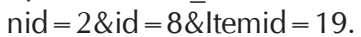

14. Instituto Nacional de Estadística y Censos. Serie histórica del Índice de Precios al Consumidor (IPC) en el Gran Buenos Aires [Internet]. INDEC [citado 12 feb 2010]. Disponible en: http://www.indec.mecon.ar/nuevaweb/cuadros/10/sh_ipc_2008.xls. 
15. Dirección Nacional de Política Macroeconómica. Información económica al día [Internet]. Ministerio de Economía y Finanzas Públicas [citado 5 feb 2010]. Disponible en: http://www.mecon.gov.ar/peconomica/basehome/infoeco.html.

16. Instituto Nacional de Estadística y Censos. Tasa de actividad, empleo, desocupación y subocupación por regiones y aglomerados urbanos desde el primer trimestre de 2003 en adelante [Internet]. INDEC [citado 10 feb 2010]. Disponible en: http://www.indec.mecon.ar/nuevaweb/cuadros/4/sh_eph_continuatrimestral.xls.

17. Suaya JA, Shepard DS, Beatty ME. Dengue burden of disease and cost of illness, TDR/SWG/08. Geneva: World Health Organization on behalf of the Special Programme for Research and Training in Tropical Diseases; 2007.

18. Duarte HH, Franca EB. Data quality of dengue epidemiological surveillance in Belo Horizonte, Southeastern Brazil. Revista de Saúde Pública. 2006;40:134-142.
19. Rigau-Pérez JG. Surveillance for an emerging disease: dengue hemorrhagic fever in Puerto Rico, 1988-1997. Puerto Rico Health Science Journal. 1999;18:337-345.

20. Meltzer MI, Rigau-Pérez JG, Clark GG, Reiter P, Gubler DJ. Using disability-adjusted life years to assess the economic impact of dengue in Puerto Rico: 1984-1994. American Journal of Tropical Medicine and Hygiene. 1998;59:265-271.

21. Armien B, Suaya JA, Quiroz E, Sah BK, Bayard V, Marchena L, Campos C, Shepard DS. Clinical characteristics and national economic cost of the 2005 dengue epidemic in Panama. American Journal of Tropical Medicine and Hygiene. 2008;79:364-371.

\section{FORMA DE CITAR}

Tarragona S, Monteverde M, Marchioni S, Caporale J, Pereiro AC, Palacios JM. Dengue en la Argentina: un análisis económico del impacto de la epidemia de 2009. Salud Colectiva. 2012;8(2):151-162.

Recibido el 13 de febrero de 2012

Versión final presentada el 23 de abril de 2012

Aprobado el 16 de mayo de 2012 